\title{
Longitudinal Findings of MRI and PET in West Syndrome with Subtle Focal Cortical Dysplasia
}

(D). Sakaguchi, DH. Kidokoro, (D). Ogawa, (D). Okai, (D). Ito, (D). Yamamoto, (D) A. Ohno, DT. Nakata, (D). Tsuji, (D)T. Nakane, (D) H. Kawai, (D) K. Kato, DS. Naganawa, and (D). Natsume

\begin{abstract}
BACKGROUND AND PURPOSE: Despite the development of neuroimaging, identification of focal cortical dysplasia remains challenging. The purpose of this study was to show the longitudinal changes of MR imaging and FDG-PET in patients with West syndrome and subtle focal cortical dysplasia.
\end{abstract}

MATERIALS AND METHODS: Among 52 consecutive patients with West syndrome, 4 were diagnosed with subtle focal cortical dysplasia on 3T MR imaging. MR imaging and PET findings were evaluated longitudinally at onset and at 12 and 24 months of age.

RESULTS: At the onset of West syndrome, MR imaging demonstrated focal signal abnormalities of the subcortical white matter in 2 patients. In the other 2 patients, focal subcortical high-intensity signals became visible on follow-up T2WI as myelination progressed. PET at onset showed focal cortical hypometabolism in 3 patients, with 1 of these patients also having focal hypermetabolism and 1 having normal findings. On PET at 24 months, hypometabolism persisted in 2 patients and disappeared in 1, and hypermetabolism disappeared in 1. In 1 patient with normal MR imaging and PET findings at onset, focal hyperintensity and hypometabolism first appeared at 24 months of age. The findings on MR imaging and PET in these patients evolved differently with brain maturation and the clinical course.

CONCLUSIONS: Subtle focal cortical dysplasia can be undetectable on MR imaging the onset of West syndrome and is not always accompanied by hypometabolism or hypermetabolism on PET. Longitudinal MR imaging and PET studies may be useful for detecting such lesions. Even in West syndrome with a congenital structural abnormality, PET findings evolve differently with brain maturation and the clinical condition.

ABBREVIATIONS: EEG = electroencephalography; $F C D=$ focal cortical dysplasia; $W S=$ West syndrome

W est syndrome (WS) is an age-dependent epileptic encephalopathy characterized by a triad of epileptic spasms, hypsarrhythmia on electroencephalography (EEG), and neurodevelopmental regression. WS is attributed to various etiologies, but

\footnotetext{
Received April 23, 2018; accepted after revision July 8.

From the Departments of Pediatrics (Y.S., H. Kidokoro, C.O., Y.O., Y.I., H.Y., A.O., T. Nakata, J.N.), Radiology (T. Nakane, H. Kawai, S.N.), Radiological and Medical Laboratory Sciences (K.K.), and Developmental Disability Medicine (J.N.), Nagoya University Graduate School of Medicine, Nagoya, Japan; Brain and Mind Research Center (H. Kidokoro, Y.I., H.Y., H. Kawai, S.N., J.N.), Nagoya University, Nagoya, Japan; and Department of Pediatrics (T.T.), Okazaki City Hospital, Okazaki, Japan.

This work was supported by a Grant-in-Aid for Scientific Research (C) 23591492 and a Grant-in-Aid for Scientific Research on Innovative Areas (Comprehensive Brain Science Network) from the Ministry of Education, Culture, Sports, Science and Technology of Japan.

Please address correspondence to Jun Natsume, MD, PhD, Department of Pediatrics, Nagoya University Graduate School of Medicine, 65 Tsurumai-cho, Showa-ku, Nagoya, Aichi 466-8550, Japan; e-mail: junnatsu@med.nagoya-u.ac.jp

- Indicates open access to non-subscribers at www.ajnr.org

E Indicates article with supplemental on-line tables.

7 Indicates article with supplemental on-line photo.

http://dx.doi.org/10.3174/ajnr.A5772
}

the cause is undetermined in $20 \%$ of patients. ${ }^{1}$ The presumed etiology in such cases includes genetic abnormalities and focal cortical dysplasia (FCD).

Despite recent developments in neuroimaging techniques, identification of FCD remains challenging. Subtle FCD can be missed on MR imaging at the onset of WS. ${ }^{2}$ It has also been reported that MR imaging findings of subtle FCD can disappear with white matter maturation. ${ }^{3}$ FDG-PET may suggest FCD even if initial MR imaging findings are normal. ${ }^{4}$ However, the findings of PET at onset evolve along with the patient's epilepsy. ${ }^{5-9} \mathrm{On}$ MR imaging evaluation of infants, the status of myelination affects the findings, and MR imaging findings of FCD cannot be detected or differ from those in older patients. ${ }^{2}$ Thus, longitudinal MR imaging and PET studies may be useful to diagnose FCD accurately.

We hypothesized that serial evaluation of MR imaging and PET from the onset to 2 years of age shows the evolution of subtle FCD findings and leads to the accurate diagnosis and location of the lesions in patients with WS. To verify this hy- 
pothesis, we reviewed our series of patients with WS in whom MR imaging and PET were longitudinally performed during 2007 and 2013.

\section{MATERIALS AND METHODS}

This study was approved by the Research Ethics Committee at Nagoya University Graduate School of Medicine. Informed consent was obtained from patients who participated in clinical investigations.

\section{Patient Selection}

Between 2007 and 2013, fifty-two children admitted to Nagoya University Hospital were newly diagnosed as having WS. In a systematic review of these 52 patients, 23 were diagnosed as having an unknown etiology at the onset of WS, meeting the following criteria (previously termed "cryptogenic"): 1) normal birth and absence of any etiologic factors related to WS; 2) normal development before onset and absence of neurologic abnormalities at onset; 3 ) the occurrence of clusters of spasms without any other types of seizures before the onset of spasms; and 4) normal laboratory and MR imaging findings at onset. Among the 23 patients with an unknown etiology at onset, 2 patients were later diagnosed as having FCD on MR imaging. In the remaining 29 patients with genetic, structural, or metabolic etiologies, 2 patients had subtle focal abnormalities of FCD on 3T MR imaging at onset. Two other patients had diffuse or bilateral FCD that was clearly recognized on MR imaging. These 2 patients were not included in this study because the focus was on patients with focal, subtle lesions that can be missed on conventional MR imaging. Thus, the present study included 4 patients in whom subtle FCD was seen at onset or during the follow-up period.

\section{Neuroimaging Protocol}

MR Imaging. MR imaging was performed longitudinally at the diagnosis of WS before adrenocorticotropic hormone therapy and at 12 and 24 months of age. Additional MR imaging was performed according to clinical need. MR imaging was performed using a 3T scanner (Magnetom Trio, a Tim System; Siemens, Erlangen, Germany) with a 32-channel phased array head coil. All patients were sedated with oral chloral hydrate $(80 \mathrm{mg} / \mathrm{kg})$ before the examination. When patients did not appear sufficiently sedated after chloral hydrate intake, intravenous midazolam or ketamine was administered. We acquired the following images: axial T1WI (TR/TE, $400 / 11$ or $700 / 6.8 \mathrm{~ms}$; slice thickness, $5 \mathrm{~mm}$ ); T2WI (TR/TE, 5210/72 or 5200/131 ms; slice thickness, $5 \mathrm{~mm}$ ); FLAIR images (TR/TE, 9000/139 ms; TI, 2500 ms; slice thickness, $5 \mathrm{~mm}$ ); sagittal T1WI (TR/TE, 500/6.8 ms; slice thickness, $5 \mathrm{~mm}$ ); coronal T2WI (TR/TE, 5210/72 or 5210/131 ms; slice thickness, $5 \mathrm{~mm}$ ); and T1-weighted sagittal MPRAGE (TR/TE, 1570/2.2 ms; slice thickness, $1 \mathrm{~mm}$ ) with reconstructed axial and coronal images.

FDG-PET. FDG-PET was performed as part of the clinical routine to search for underlying pathologies using a Headtome $\mathrm{V}$ scanner (Shimadzu, Kyoto, Japan). All patients were sedated with a chloral hydrate suppository $(50 \mathrm{mg} / \mathrm{kg})$ before the PET examination. When patients did not appear sufficiently sedated after chloral hydrate, intravenous midazolam or ketamine was administered 45 minutes after the FDG injection. Patients were scanned for 60 minutes after intravenous administration of FDG. EEGs were not monitored during the PET scans. Instead, pediatric neurologists observed all patients throughout the scan and confirmed that there were no seizure manifestations during the examination. Thirty-four axial images (4-mm-thick) were obtained, and the images from 45 to 60 minutes after FDG injection were used for the evaluation.

\section{Assessment of MR Imaging and PET Findings}

MR imaging and PET findings were assessed with visual inspection by 3 pediatric neurologists (J.N., Y.S., and H. Kidokoro) and 2 radiologists (T. Nakane for MR imaging and K.K. for PET). One of the radiologists was a pediatric neuroradiologist (T. Nakane) regularly involved in the MR imaging evaluation of patients with epilepsy. Another radiologist (K.K.) specialized in nuclear medicine and evaluating PET findings of patients with epilepsy. MR imaging was independently evaluated by 3 pediatric neurologists (J.N., Y.S., and H. Kidokoro) and 1 neuroradiologist (T. Nakane). PET was evaluated independently by 3 pediatric neurologists (J.N., Y.S., and H. Kidokoro) and 1 radiologist specialized in nuclear medicine (K.K.). Four reviewers agreed on the presence or absence of abnormal findings on MR imaging or PET, and they decided on the distribution of the abnormalities by discussion. On MR imaging, whether there were structural abnormalities, abnormal high or low intensity signals, and delayed myelination was evaluated. On PET, focal hypometabolism or hypermetabolism was defined as a regional decrease or increase, respectively, in FDG accumulation in $\geq 2$ gyri on $\geq 2$ slices. To assess localization of PET abnormalities, we performed an MR imaging-PET coregistration technique using SPM12b software (http://www.fil.ion. ucl.ac.uk/spm/software/spm12) and the image visualization software Register (Brain Imaging Center, Montreal Neurological Institute, Montreal, Quebec, Canada).

\section{Clinical Data}

Other clinical variables were collected from medical records. EEG recordings were performed at onset, during adrenocorticotropic hormone therapy, and at least at 12 and 24 months of age. The EEG records were assessed by 3 pediatric neurologists (J.N., Y.S., and H. Kidokoro). All patients with WS were followed by pediatric neurologists at our outpatient clinics until 5-7 years of age. The developmental quotient or intelligence quotient was assessed at the last follow-up using the Kinder Infant Development Scale, a developmental test standardized in Japan in 1989. ${ }^{10}$ If the patient was older than 7 years of age, the Wechsler Intelligence Scale for Children, Fourth Edition, was administered because the Kinder Infant Development Scale was appropriate for children younger than 7 years of age. Developmental status was considered as follows: 1) normal, when patients had a developmental quotient of $\geq 80$; 2) borderline, when patients had a developmental quotient between 70 and $79 ; 3)$ mild delay, when patients had a developmental quotient between 50 and 69; and 4) severe delay, when patients had a developmental quotient of $<50$. 


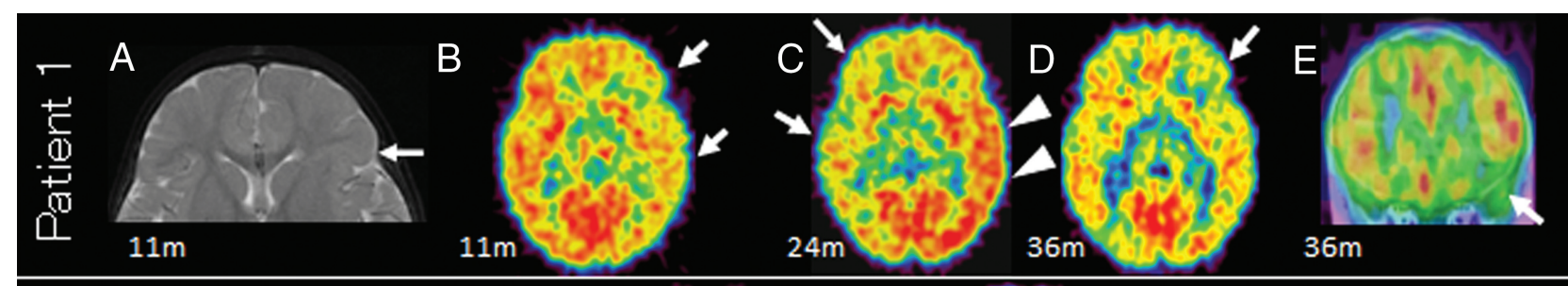

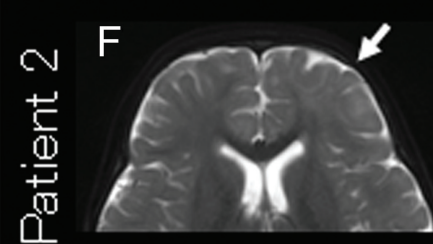

$19 \mathrm{~m}$
$\mathrm{G}$

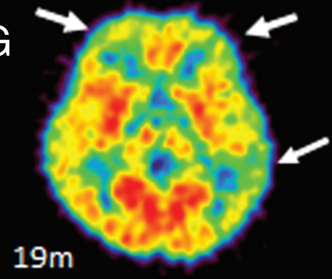

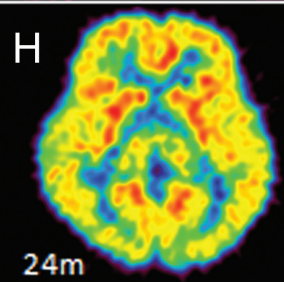
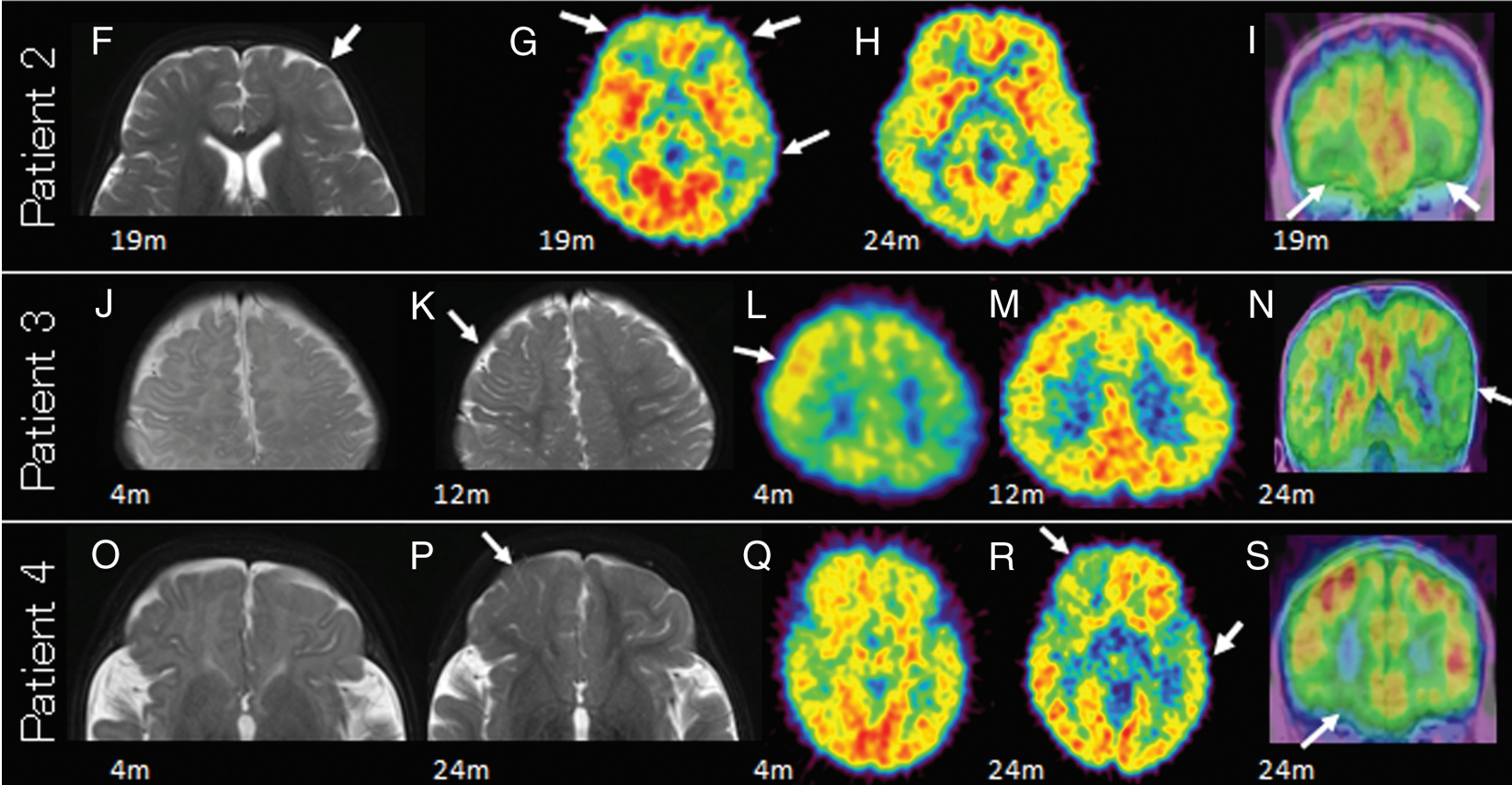

Q
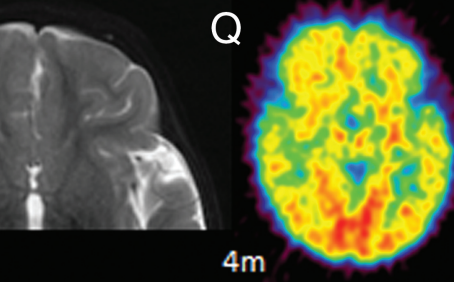

$\mathrm{R}$

$2 \mathrm{~m}=\mathrm{cos}$
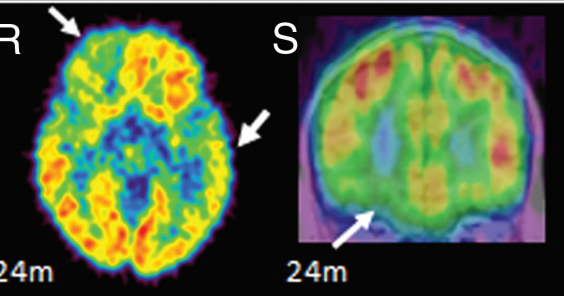

FIGURE. MR imaging and PET findings of 4 patients. Longitudinal findings of T2-weighted MR imaging and PET of patient 1 (upper row, $A-E$ ), patient 2 (second row, $F-l$ ), patient 3 (third row, $J-N$ ), and patient 4 (lower row, $O-S$ ). Patient 1: note a focal hyperintense lesion in the subcortical white matter of the left frontal lobe on axial MR imaging at onset (arrow, $A$ ). PET hypometabolism is seen in the left frontotemporal lobe at 11 months of age (arrows, B), in the right frontotemporal lobe at 24 months of age (arrows, $C$ ), and in the left frontal lobe at 36 months of age (arrow, D). PET at 24 months of age also shows possible hypermetabolism in the left temporal lobe (arrowheads, C). A coregistered coronal image of PET on MR imaging at 36 months of age shows hypometabolism in the left orbitofrontal area that corresponds to the MR imaging lesion (arrow, E). Patient 2: a hyperintense area in the subcortical white matter of the left frontal lobe on axial MR imaging at onset (arrow, F). Multifocal hypometabolism in the frontal and temporal lobes is seen on PET at onset (arrows, $G$ ) that disappears at 24 months of age $(H)$. No PET hypometabolism at 24 months of age $(H)$ is seen. Hypometabolism in the left frontal lobe corresponds to the MR imaging lesion on a coronal MR imaging-PET coregistration image (arrows, I). Patient 3: normal MR imaging findings at onset ()). Hyperintense subcortical white matter is seen in the right precentral area on MR imaging at 12 months of age (arrow, $K$ ). Left parietotemporal hypometabolism and right frontal hypermetabolism on PET is seen at onset (arrow, L). Right frontal hypermetabolism disappears at 12 months of age (M). Left temporal hypometabolism is seen on PET at 24 months of age, whereas MR imaging shows no abnormality (arrow, N). Patient 4: both MR imaging $(O)$ and PET $(Q)$ findings are normal at onset. A hyperintense area in the right orbitofrontal white matter is seen on axial MR imaging at 24 months of age (arrow, $P$ ). Hypometabolism in the right orbitofrontal lobe corresponds to the MR imaging lesion (arrow, $R$ and $S$ ) and is seen in the left temporal lobe on PET (arrows, $R$ ).

\section{RESULTS}

The profiles of the 4 patients are summarized in On-line Table 1. The age at onset of spasms ranged from 2 to 18 months. The age at diagnosis of WS ranged from 4 to 19 months. All patients had normal development before the onset of spasms and normal neurologic examination findings at onset. All patients received adrenocorticotropic hormone therapy. After adrenocorticotropic hormone therapy, 3 patients were free of seizures. Another one (patient 1) had a relapse of spasms at 17 months of age, and oral antiepileptic drugs were administered. The patient became free of seizures from 2 years of age. The developmental quotient or intelligence quotient at the last follow-up was normal in 3 patients and mildly delayed in 1 patient.

\section{MR Imaging and PET Findings}

MR imaging, PET, and EEG findings are summarized in On-line Table 2. The initial PET scans at the onset of WS showed focal cortical hypometabolism in 3 patients, and one of them (patient 3) also had focal hypermetabolism. The longitudinal findings in each patient are detailed below (Figure and On-line Figs 1-4).

Patient 1. This patient began having epileptic spasms at 7 months of age. Her initial MR imaging at 11 months of age showed a focal hyperintense lesion on both T1WI and T2WI in the left frontal white matter at the level of the foramen of Monro (Fig 1A). She underwent gadolinium-enhanced MR imaging and methionine PET to rule out a brain tumor, and both had negative findings. FDG-PET at onset showed hypometabolism in the left frontotemporal area (Fig $1 B)$, which was more widespread than the lesion on MR imaging. At 24 months of age, PET showed hypometabolism in the right frontotemporal lobe (arrows) contralateral to the MR imaging lesion and possible hypermetabolism in the left temporal lobe (arrowheads) (Fig 1C). At 36 months of age, the area of hypometabolism was localized to the left frontal lobe overlying 
the MR imaging lesion (Fig $1 D,-E$ ). The EEG at 24 months of age showed repetitive focal spikes, polyspikes, and slow waves in the left frontal area, concordant with the MR imaging lesion. The paroxysmal epileptic discharges were less frequent at 36 months of age. At the last follow-up at 5 years of age, the patient was free of seizures with antiepileptic drugs (levetiracetam and topiramate). She had mild a developmental delay. Her developmental quotient by the Kinder Infant Development Scale at 5 years of age was 57 .

Patient 2. The patient had late-onset spasms at 18 months of age, with otherwise no previous medical history of neurological abnormalities. T2WI at onset showed slight hyperintensity in the left frontal white matter (Fig $1 F$ ). PET at onset showed multifocal hypometabolism in the left frontal, left temporal, and right frontal areas (Fig $1 G,-I$ ). At 24 months of age, PET showed no abnormality, even at the site of the MR imaging lesion (Fig $1 H$ ). EEG at 24 months of age showed sporadic spikes, polyspikes, and sharp waves in the left frontal area, concordant with the MR imaging lesion. At the last follow-up at 5 years of age, the patient was free of seizures without antiepileptic drugs, except for febrile seizures. Her psychomotor development was normal. Her developmental quotient by the Kinder Infant Development Scale at 5 years of age was 95 .

Patient 3. The patient had onset of spasms at 2 months of age. MR imaging findings at onset were normal (Fig $1 J$ ), but MR imaging at 12 months of age showed a hyperintense area in the right frontal subcortical white matter (Fig $1 K$ ). The hyperintense lesion was more clearly recognized at 24 months of age with progress of myelination in the surrounding white matter. PET at 4 months of age showed left parietotemporal hypometabolism and right frontal hypermetabolism (Fig $1 \mathrm{~L}$ ). At 12 and 24 months of age, hypermetabolism in the right frontal lobe disappeared (Fig 1M), while PET showed left temporal hypometabolism and MR imaging showed no abnormality (Fig $1 N$ ). The EEG at 24 months of age showed sporadic polyspikes in the left temporal lobe that were concordant with PET hypometabolism. At the last follow-up at 7 years of age, the patient was free of seizures without antiepileptic drugs. His intelligence quotient by the Wechsler Intelligence Scale for Children, Fourth Edition, was 84. His intelligence level was within normal limits, but he had an attention deficit/hyperactivity disorder.

Patient 4. The patient began having spasms at 3 months of age. She had no abnormalities on both MR imaging and PET at 4 and 12 months of age (Fig 1O, -Q). The right orbitofrontal MR imaging lesion and hypometabolism became evident at 24 months of age (Fig $1 P,-R,-S$ ). PET at 24 months of age showed hypometabolism in the left temporal lobe at a different site from the MR imaging lesion (Fig 1R). EEG at 24 months of age showed sharp waves in the right frontal and left temporal areas that were concordant with 2 areas of PET hypometabolism. At the last follow-up at 5 years of age, the patient was free of seizures without antiepileptic drugs. She had normal psychomotor development. Her developmental quotient by the Kinder Infant Development Scale was 91 at 5 years of age.

\section{DISCUSSION}

Recent developments in neuroimaging technology have made it possible to understand the causes of WS, such as FCD. However, it is still difficult to accurately diagnose microscopic lesions.

In the present study, longitudinal MR imaging and PET findings of patients with WS and subtle MR imaging lesions of FCD are presented. In 2 cases (patients 3 and 4), no MR imaging abnormality was visible in the early infantile period, but an MR imaging abnormality became visible later with progress of myelination in the surrounding white matter. PET showed additional regional hypometabolism remote from the MR imaging lesions. In patient 3, PET at the onset of WS also showed hypermetabolism in the MR imaging lesion that was visible later at 12 months of age.

Two of the 4 patients had negative MR imaging findings in the early infantile period, and they were regarded as having WS of unknown etiology at onset. Abnormal white matter hyperintensity became visible on the T2-weighted follow-up MR imaging. Signal change of the white matter on MR imaging occurs during the first 2 years of life as a result of myelination. Myelination is recognized as a high signal on T1WI and a low signal on T2WI. In general, myelination progresses from caudal to cephalad and from dorsal to ventral. ${ }^{11}$ Therefore, the subcortical white matter matured last (other than the in calcarine and Rolandic areas), proceeding from the occipital region anteriorly to the anterior frontal and temporal lobes. Changes of myelination on T2WI appear at 14-18 months of age in the midfrontal subcortical white matter and at 24-30 months of age in the anterior frontal subcortical white matter. ${ }^{11}$ In the present study, the lesion in the precentral area (patient 3 ) became visible at 12 months of age, and the lesion in the orbitofrontal area (patient 4) became visible at 24 months of age. The age at detection of the lesion on MR imaging matched the period of myelination. Before the maturational change of subcortical white matter, it is difficult to detect the characteristic findings of FCD, such as blurring of the gray-white matter junction and signal changes in subcortical white matter. ${ }^{12,13}$ Thus, all patients with WS of unknown etiology at diagnosis should be re-evaluated by MR imaging at 18-24 months of age.

Concerning FDG-PET findings, it has been reported that maturational change occurs in regional cerebral glucose metabolism, especially during the infantile period. ${ }^{14,15}$ During this period, the cerebral glucose uptake pattern progresses from the subcortical gray matter to the cortex and from posterior-to-anterior cortical areas. ${ }^{14,15}$ In early infancy, PET shows lower uptake in the frontal and temporal lobes than in the parietal and occipital lobes, and frontotemporal pathologic hypometabolism can be missed in this period. In the present study, the frontal hypometabolism at onset in patient 4 was unclear, possibly due to the nature of the glucose metabolism pattern in the infantile period.

FDG-PET is useful for detecting FCD in WS. A previous study showed that PET was more sensitive than MR imaging for patients with a mild degree of cortical dysplasia, though MR imaging was performed using a $1.5 \mathrm{~T}$ scanner. ${ }^{16}$ More recent reports with $1.5 \mathrm{~T}$ or 3T MR imaging scanners have also shown the usefulness of PET in patients with MR imaging negative for FCD, especially with PET/MR imaging coregistration. ${ }^{17}$ Chugani et $\mathrm{al}^{18}$ showed that 
PET hypometabolism was consistent with EEG localization of epileptogenic foci in patients with MR imaging negative for WS. Asano et $\mathrm{al}^{19}$ also reported that the ictal electrocorticographic findings associated with spasm originated within a PET hypometabolic region. In the present study, PET showed hypometabolism or hypermetabolism that corresponded to the MR imaging lesions, but also hypometabolism in areas remote from the MR imaging lesions. In 2 patients (patients 3 and 4), the remote hypometabolic areas were accompanied by focal independent epileptiform discharges on the interictal EEG. The remote hypometabolic areas with focal epileptiform discharges may indicate latent dysplastic lesions or secondary epileptogenic foci.

Even in the region of structural abnormalities such as FCD, PET findings evolve with time from hypometabolism to normal, hypermetabolism to normal, or normal to hypometabolism during the first years of life. Previous studies have demonstrated that FDG-PET showed hypometabolism in the region of FCD, usually corresponding to, but somewhat more extensive than, the MR imaging lesion. ${ }^{20}$ In contrast, cases with FCD lesions showing hypermetabolism have also been reported. ${ }^{21}$ While subclinical seizures during the scan possibly cause hypermetabolism on PET, it has been reported that hypermetabolism can be seen in regions with frequent epileptiform discharges, even in the interictal state, especially with FCD. ${ }^{22}$ While several studies have shown that PET has an advantage for detecting subtle FCD, Sankar et $\mathrm{al}^{2}$ reported that PET was not as sensitive as follow-up MR imaging in patients with WS. The present observation of normal PET findings at the site of FCD at some points is consistent with the results of Sankar et al. Conversely, this serial PET study showed hypometabolism or hypermetabolism at the site of FCD on PET scans at least at 1 point in all patients. Evaluation of serial PET with follow-up MR imaging has a complementary effect in detecting subtle lesions.

Generally, patients with WS with FCD have poor seizure control by antiepileptic drugs and are candidates for resective surgery. ${ }^{23,24}$ However, all patients in the present study had favorable seizure outcomes without surgery, at least until preschool age, and the developmental outcome was also favorable in 3 patients. This difference may be explained by the different characteristics of the MR imaging and PET findings of FCD in the present cases. Typical MR imaging findings of FCD are a focal area of cortical thickness and blurring of the gray-white matter junction, with or without signal change in subcortical white matter on T2WI and FLAIR. ${ }^{25}$ All patients in the present study, however, showed only a hyperintense signal on T2WI in the subcortical white matter, without cortical thickness or blurring of the gray-white matter junction. Additionally, PET hypometabolism was not persistently observed at the MR imaging lesion of FCD. The different neuroimaging characteristics and, probably, prompt initiation of adrenocorticotropic hormone with a short treatment lag after onset might be associated with the favorable outcomes in the present study.

The present study has some limitations. First, the diagnosis of FCD was not confirmed pathologically because the patients showed favorable seizure outcomes. The differential diagnosis of the MR imaging lesion may include benign tumor and polymicrogyria. However, none of the patients showed less cortical thickening and intravenous contrast enhancement than observed in brain tumors ${ }^{26}$ or an abnormal gyral pattern, increased cortical thickness, or irregularity of the cortical-white matter junction as seen in polymicrogyria. ${ }^{27,28}$ It has been reported that FCD has a variety of features on MR imaging studies and does not always show the characteristic signs, such as cortical thickening, blurring of the gray-to-white matter surface, and signal changes in the underlying white matter. ${ }^{29}$ From these observations, the MR imaging findings in the present study suggest FCD even without confirmation by histopathology. Second, a strong limitation of the present study is that the sample size was very small. Studies with a large number of patients are needed to draw conclusions on the usefulness of MR imaging and PET. Third, MR imaging and PET were assessed by visual inspection without using quantitative or statistical techniques (such as volumetric MR imaging analysis or quantification of glucose metabolism). Using these techniques may increase sensitivity and objectivity.

\section{CONCLUSIONS}

A case series of patients with WS with FCD who showed subtle or unrecognized MR imaging abnormalities at diagnosis was presented. The findings on MR imaging and PET in these patients evolved differently with brain maturation and the clinical course of WS. Their seizure outcomes were consistently favorable compared with previous reports. Serial MR imaging and PET studies may be useful to accurately diagnose the etiology and make the appropriate prediction of seizure outcomes in WS.

\section{REFERENCES}

1. Riikonen R. Epidemiological data of West syndrome in Finland. Brain Dev 2001;23:539-41 CrossRef Medline

2. Sankar R, Curran JG, Kevill JW, et al. Microscopic cortical dysplasia in infantile spasms: evolution of white matter abnormalities. AJNR Am J Neuroradiol 1995;16:1265-72 Medline

3. Eltze CM, Chong WK, Bhate S, et al. Taylor-type focal cortical dysplasia in infants: some MRI lesions almost disappear with maturation of myelination. Epilepsia 2005;46:1988-92 CrossRef Medline

4. Chugani HT, Shields WD, Shewmon DA, et al. Infantile spasms, I: PET identifies focal cortical dysgenesis in cryptogenic cases for surgical treatment. Ann Neurol 1990;27:406-13 CrossRef Medline

5. Itomi K, Okumura A, Negoro T, et al. Prognostic value of positron emission tomography in cryptogenic West syndrome. Dev Med Child Neurol 2002;44:107-11 CrossRef Medline

6. Maeda N, Watanabe K, Negoro T, et al. Transient focal cortical hypometabolism in idiopathic West syndrome. Pediatr Neurol 1993;9: 430-34 CrossRef Medline

7. Maeda N, Watanabe K, Negoro T, et al. Evolutional changes of cortical hypometabolism in West's syndrome. Lancet 1994;343: 1620-23 CrossRef Medline

8. Natsume J, Maeda N, Itomi K, et al. PET in infancy predicts longterm outcome during adolescence in cryptogenic West syndrome. AJNR Am J Neuroradiol 2014;35:1580 - 85 CrossRef Medline

9. Natsume J, Watanabe K, Maeda N, et al. Cortical hypometabolism and delayed myelination in West syndrome. Epilepsia 1996;37: 1180-84 CrossRef Medline

10. Aoki S, Hashimoto K, Ikeda N, et al. Comparison of the Kyoto Scale of Psychological Development 2001 with the parent-rated Kinder Infant Development Scale (KIDS). Brain Dev 2016;38:481-90 CrossRef Medline

11. Barkovich AJ, Raybaud C. Pediatric Neuroimaging. 5th ed. Philadelphia: Lippincott Williams \& Wilkins; 2012

12. Ballesteros MC, Hansen PE, Soila K. MR imaging of the developing 
human brain, Part 2: postnatal development. Radiographics 1993;13: 611-22 CrossRef Medline

13. Barkovich AJ, Kjos BO, Jackson DE Jr, et al. Normal maturation of the neonatal and infant brain: MR imaging at 1.5 T. Radiology 1988; 166:173-80 CrossRef Medline

14. Chugani HT, Phelps ME. Maturational changes in cerebral function in infants determined by $18 \mathrm{FDG}$ positron emission tomography. Science 1986;231:840-43 CrossRef Medline

15. Kinnala A, Suhonen-Polvi H, Aärimaa T, et al. Cerebral metabolic rate for glucose during the first six months of life: an FDG positron emission tomography study. Arch Dis Child Fetal Neonatal Ed 1996; 74:153-57 Medline

16. Kim SK, Na DG, Byun HS, et al. Focal cortical dysplasia: comparison of MRI and FDG-PET. J Comput Assist Tomogr 2000;24:296-302 CrossRef Medline

17. Desarnaud S, Mellerio C, Semah F, et al. 18F-FDG PET in drugresistant epilepsy due to focal cortical dysplasia type 2: additional value of electroclinical data and coregistration with MRI. Eur J Nucl Med Mol Imaging 2018;45:1449-60 CrossRef Medline

18. Chugani HT, Shewmon DA, Shields WD, et al. Surgery for intractable infantile spasms: neuroimaging perspectives. Epilepsia 1993;34: 764-71 CrossRef Medline

19. Asano E, Juhász C, Shah A, et al. Origin and propagation of epileptic spasms delineated on electrocorticography. Epilepsia 2005;46:1086-97 CrossRef Medline

20. Lee N, Radtke RA, Gray L, et al. Neuronal migration disorders: positron emission tomography correlations. Ann Neurol 1994;35:290-97 CrossRef Medline
21. Namer IJ, Valenti-Hirsch MP, Scholly J, et al. Hypermetabolism during resting-state FDG-PET suggesting intrinsic epileptogenicity in focal cortical dysplasia. Clin Nucl Med 2014;39:993-95 CrossRef Medline

22. Bansal L, Miller I, Hyslop A, et al. PET hypermetabolism in medically resistant childhood epilepsy: incidence, associations, and surgical outcome. Epilepsia 2016;57:436-44 CrossRef Medline

23. Kang JW, Rhie SK, Yu R, et al. Seizure outcome of infantile spasms with focal cortical dysplasia. Brain Dev 2013;35:816-20 CrossRef Medline

24. Yum MS, Ko TS, Lee JK, et al. Surgical treatment for localizationrelated infantile spasms: excellent long-term outcomes. Clin Neurol Neurosurg 2011;113:213-17 CrossRef Medline

25. Widdess-Walsh P, Diehl B, Najm I. Neuroimaging of focal cortical dysplasia. J Neuroimaging 2006;16:185-96 CrossRef Medline

26. Bronen RA, Vives KP, Kim JH, et al. Focal cortical dysplasia of Taylor, balloon cell subtype: MR differentiation from low-grade tumors. AJNR Am J Neuroradiol 1997;18:1141-51 Medline

27. Battal B, Ince $S$, Akgun $V$, et al. Malformations of cortical development: $3 \mathrm{~T}$ magnetic resonance imaging features. World $\mathrm{J} \mathrm{Ra}$ diol 2015;7:329-35 CrossRef Medline

28. Leventer RJ, Guerrini R, Dobyns WB. Malformations of cortical development and epilepsy. Dialogues Clin Neurosci 2008;10:47-62 Medline

29. Bast T, Ramantani G, Seitz A, et al. Focal cortical dysplasia: prevalence, clinical presentation and epilepsy in children and adults. Acta Neurol Scand 2006;113:72-81 CrossRef Medline 